\title{
Mental effort and task interference in auditory attention
}

\author{
RUTH KIMCHI \\ University of California, Berkeley, California
}

\begin{abstract}
Dual-task performance was examined in three experiments. The primary task was to repeat or to add one to four digits presented auditorily at a rate of 1 digit/second. This primary task was combined with three different secondary tasks in which subjects listened to a list of words either for later recognition of some of the words (Experiment 1) or for detection and a later report of a target word (Experiments 2 and 3). Different patterns of task interference were obtained. Recognition performance was sensitive to between-task variations in capacity demands but did not reflect momentary attentional demands within the primary task. Detection performance reflected both between-task and within-task variation in capacity demands of the primary task. The interference between the primary and the secondary task was mutual, with more interference when the selection-cue in the detection task was a category name than when it was the target word itself. These findings are discussed in terms of effort theory of attention and the role of attentional strategies in dual-task performance.
\end{abstract}

A person cannot easily perform two simultaneous tasks. "Bottleneck"' theories of attention (e.g., Broadbent, 1958, 1971; Deutsch \& Deutsch, 1963, 1967; Keele, 1973; Treisman, 1969) have posited a particular stage of processing (either earlier or later in the information-processing sequence) at which the limitation occurs. An alternative to structural theories of attention is capacity theories, which allow more flexibility in allocation of attention (e.g., Kahneman, 1973; Moray, 1967; Navon \& Gopher, 1979, 1980; Norman \& Bobrow, 1975; Posner \& Snyder, 1975; Wickens, 1980). The human processing system is assumed to possess some limited psychological resources or capacity which can be allocated to different activities as required by task demands. The allocation of capacity is assumed to be under some cognitive control.

An elaborated capacity theory of attention has been proposed by Kahneman (1973), who identifies attention with a general pool of limited capacity or "mental effort." Effort or attention is controlled by task demands. The model assumes a feedback mechanism by which an evaluation of momentary demands and performance controls arousal and thereby the supply of effort for the successful continuation of that performance. Interference between concurrent tasks occurs when their combined demands exceed the capacity limit. In addition to this non-

This paper is based on a master's thesis completed under the direction of Daniel Kahneman and submitted to the Hebrew University of Jerusalem. I wish to thank Daniel Kahneman for his guidance and encouragement throughout the project, and Daniel Morrow and Steve Palmer for helpful comments and suggestions on this manuscript. Requests for reprints should be sent to Rutie Kimchi, Center for Human Information Processing C-009, University of California, San Diego, La Jolla, California 92093. specific capacity interference, an all-or-none type of interference due to structural limitations is possible. Dividing attention between simultaneous tasks is done according to allocation policy, which is influenced by internal and external factors such as arousal, evaluation of demands, and payoffs.

Several theorists have suggested that the processing system can be viewed as being composed of a number of mechanisms, each having its own capacity which can be distributed among several processes (e.g., Navon \& Gopher, 1979, 1980; Wickens, 1980). In such a multiple resources model, interference between concurrent activities occurs when the demands for capacity from any of the multiple pools of capacity exceed the capacity limit in each pool.

The present research focuses on the predictions of the capacity (either general or multiple) model concerning interference between concurrent activities. According to capacity theory, the extent of such interference can be predicted by considering the demands these activities impose on a common pool of effort or capacity. Judgments about the amount of effort that a particular task requires have been made either by appealing to widely shared intuitions or, empirically, by examining the effect of one task on another task in a dual-task situation (e.g., the secondary-task technique; see Kerr, 1973, for a review). Increasing processing load (i.e., demands for capacity or effort) of one task is supposed to affect performance on the concurrent task as the subject reallocates resources to deal with the change in the demand. However, the effect of processing load can be confounded with the effect of allocation policies of subjects, and several researchers have suggested the joint manipulation of task emphasis and task difficulty to separate the two effects (see 
Navon \& Gopher, 1979, 1980, and Wickens, 1980, for a detailed discussion).

In the present series of experiments, interference in dual-task conditions was examined by employing a primary task for which several independent measures of capacity requirements already existed from previous research. Of particular importance is the task-evoked pupillary response, which seems to be a reliable measure of mental effort: It provides a reliable and sensitive indication of within-task and between-task variation in processing load (see Beatty, 1982, for an extensive review).

The task involved listening to four spoken digits presented to the right ear and responding with four digits. In the "repeat" task, the subjects merely repeated the four digits they had heard, and in the "transform" task, they added 1 to each digit. It has been found that a characteristic pattern of autonomic responses accompanies the performance of these tasks. The diameter of the pupil, heart rate, and skin conductance all increase markedly during the listening phase, reaching a peak early in the response phase and decreasing thereafter. The peak response in each measure was higher in the transform task than in the repeat task (Kahneman \& Beatty, 1966; Kahneman, Tursky, Shapiro, \& Crider, 1969). The transform task was combined with a visual detection task in a dual-task situation (Kahneman, 1970; Kahneman, Beatty, \& Pollack, 1967). Results have shown that parallel to these autonomic changes are changes in the subject's ability to detect a briefly presented visual target. In the present experiments, the transform and the repeat tasks were combined with three different secondary tasks, all involving the subject's listening to a list of words presented to the left ear and requiring either (1) later recognition of some of the words or (2) detection and a later report of a target word. Instructions and a payoff matrix emphasized the primary task over the secondary task. The particular predictions of the effort theory are that there will be more interference from the transform task than from the repeat task to the secondary task, and that interference from the primary task will vary along an inverted- $U$ curve as a function of time, such that interference will be greatest in the middle of the task. Confirmation of these predictions will provide further converging evidence for the concept of mental effort.

\section{EXPERIMENT 1}

\section{Method}

Sabjects. Twelve students at the Hebrew University of Jerusalem, nine females and three males aged 20-27 years, participated in the experiment. All subjects were native speakers of Hebrew, were right-handed, and had no hearing problems.

Primary task. The primary task involved listening to four digits spoken in Hebrew, and responding with four digits. In the repeat task, the subject was required to repeat the digits as presented; in the transform task, the subject was required to add 1 to each digit (e.g., 3519 becomes 4620 ). The series of digits were constructed so that each series contained four different randomly chosen digits. The digits were presented to the subject's right ear at a constant rate of $1 \mathrm{digit} / \mathrm{sec}$, and were accompanied by metronome beats. Each trial consisted of 13 beats; the word "ready" was presented on the third beat, then four digits were presented on the following four beats, followed by the word "answer," and the response was given on the following four beats. Cases in which the subject missed the rhythm were treated as failures in the primary task.

Secondary task. The secondary task involved the presentation of 11 bisyllabic Hebrew words to the subject's left ear on the beats of the metronome, starting from the word "ready" and ending 1 sec after the time at which the subject was instructed to say the last digit of his response. The word series were constructed from a pool of bisyllabic words drawn from Balgur (1968). Immediately after the completion of the primary task, but only if that task had been successfully performed, the subject was given a recognition list which included 10 words, of which 5 were the words presented simultaneously with the primary-task stimuli and 5 were distractors drawn from the same pool of bisyllabic words. Only the words that had been presented simultaneously with the first and third digits of the listening phase, with the word "answer," and with the second and last digits of the response phase of the primary task were probed in the recognition test, but the subjects were not informed of this. The subjects were instructed to mark down the words they believed had appeared in the leftear message. They could choose as many words as they wished, but were told that only 5 of the 10 words had actually appeared on the message.

Payoff matrix. The subjects were paid .06 Israeli pounds for a correct performance on the primary task and .01 Israeli pounds for each correct recognition; they were penalized .01 Israeli pounds for marking a word that had not been presented. If subjects failed the primary task, they were given no chance to perform the recognition task.

Design and Procedure. The experiment employed a completely within-subjects, three-factor design: condition (dual-task and control), task (repeat and transform), and time of occurrence of critical items. On dual-task trials, the subjects performed both the primary and the secondary tasks. There were two types of control trials: (1) primary-task control trials, in which subjects performed only the primary task and ignored the list of words in the left ear, and (2) secondary-task control trials, in which the secondary task was the important one and the transform task was made very simple-the subjects heard 1111 and responded 2222 . Note that the control conditions also involved dichotic presentation identical to this in the dual-task conditions (i.e., the digits were presented to the subject's right ear and the list of words to the subject's left ear). Thus, the baseline performance on each task took into account any effect of differential ear advantage.

Each subject performed a total of 164 trials, consisting of 24 practice trials and 140 experimental trials. The experimental trials were arranged in 20 blocks of 7 trials each-5 dual-task trials, 1 primary-task control trial, and 1 secondary-task control trial. The dual-task trials as well as the primary-task control trial in each block were all either the repeat or the transform task; the task was alternated in successive blocks. The order of the series of digits and words was the same for all subjects, but different subjects were assigned different sequences of tasks, so that each series was associated with each task equally often across subjects. Prior to each trial, the subject was instructed about the nature of the trial. An "add 0" (the repeat task) and an "add 1" (the transform task) instruction indicated dual-task trials; the instruction "without words" indicated a primary-task control trial, and the instruction "without digits" indicated a secondary-task control trial.

The subjects served individually in a quiet room equipped with a tape recorder and a set of earphones. The instructions, which explained the primary and the secondary tasks and the payoffs, were presented to the subject's right ear. 


\section{Results and Discussion}

Performance on the primary task. Mean percentages of success on the primary task are presented in Table 1. A three-way analysis of variance (task $x$ condition $\times$ subjects) indicated a significant effect of task (repeat/transform) $[F(1,11)=42.86, p<.001]$. The difference between performance on the primary task in the dual-task condition and that in the primary-task control condition $[F(1,11)=3.93$, .05 $<\mathrm{p}<.10]$ and the interaction between task and condition $[F(1,11)=3.29, .05<p<.10]$ just approached significance.

Performance on the secondary task. Probabilities of failure in recognition as a function of the time of occurrence of the words are presented in Figure 1. There was no significant difference between the false recognition rate in the various conditions, but since the false recognition rate in the secondary-task control condition $(15 \%)$ tended to be higher than in the dual-task conditions (13\% in both), the recognition data were submitted to a signal detection analysis. A d' value based on hits and false alarms was calculated for each subject under each of the condition $x$ time combinations, and a three-way analysis of variance (condition $\times$ time $\times$ subjects) of these $d$ ' values was performed. The effect of condition $[F(2,22)=5.85, p<.01]$, the effect of time $[F(4,44)$ $=111.68, \mathrm{p}<.001]$, and the interaction between condition and time $[F(8,88)=3.09, p<.01]$ were significant.

Newman-Keuls tests indicated that memory for the left-ear message was lower when the subjects performed the transform task than when they performed the repeat task $[q(2,11)=5.40, p<.01]$, and that, in both dual-task conditions, it was lower than in the control condition $[q(3,11)=10.84, p<.01$, for the transform task; $q(2,11)=5.44, p<.01$, for the repeat task]. These results suggest that both the repeat and the transform task required processing capacity, and that the transformation imposed a greater load on the system.

Table 1

Mean Percentages of Success on the Primary Task for Each Condition of Each Experiment

\begin{tabular}{|c|c|c|}
\hline \multirow[b]{2}{*}{ Condition } & \multicolumn{2}{|c|}{ Task } \\
\hline & Repeat & Transform \\
\hline & \multicolumn{2}{|c|}{ Experiment $1(n=12)$} \\
\hline \multirow[t]{2}{*}{$\begin{array}{l}\text { Dual Task } \\
\text { Control }\end{array}$} & $\begin{array}{l}95.00 \\
95.53\end{array}$ & $\begin{array}{l}74.17 \\
81.17\end{array}$ \\
\hline & \multicolumn{2}{|c|}{ Experiment $2(n=16)$} \\
\hline \multirow[t]{2}{*}{$\begin{array}{l}\text { Dual Task } \\
\text { Control }\end{array}$} & $\begin{array}{l}92.80 \\
96.25\end{array}$ & $\begin{array}{l}70.13 \\
75.63\end{array}$ \\
\hline & \multicolumn{2}{|c|}{ Experiment $3(n=12)$} \\
\hline $\begin{array}{l}\text { Dual Task } \\
\text { Control }\end{array}$ & $\begin{array}{l}87.17 \\
95.00\end{array}$ & $\begin{array}{l}60.00 \\
70.83\end{array}$ \\
\hline
\end{tabular}

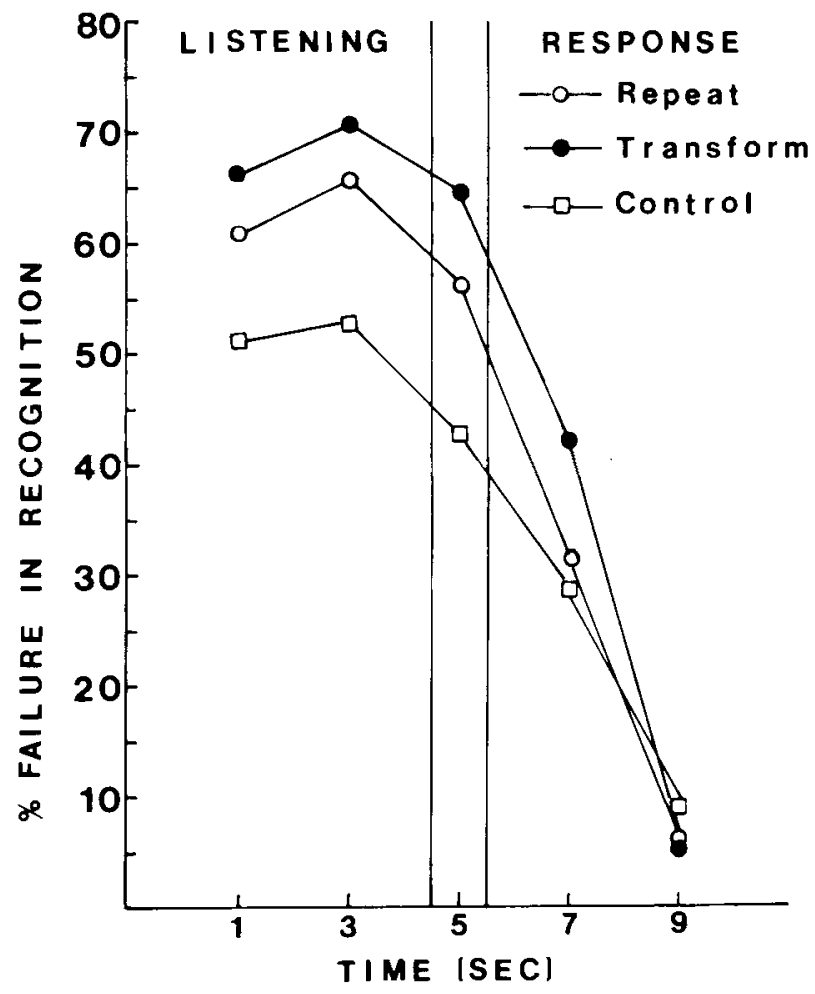

Figure 1. Results of Experiment 1: Probability of fallure in recognition as a function of time of presentation of the words.

The hypothesis that the distribution of failure in recognition in the dual-task conditions had an inverted- $U$ curve was tested by trend analysis. In order to control for the possibility that the distribution of failure on the secondary task was not due to interference from the primary task, the analysis was performed on the following scores: For each subject, the difference between percentage of failure on the dual-task condition and the secondary-task control condition was calculated. Hereafter, I will refer to these scores as the "difference scores." The quadratic regression did not turn significant $[F<1$ for the repeat task; $F(1,11)=1.365, p>.25$, for the transform task].

Thus, recognition performance was sensitive to the different processing demands of the two primary tasks, but it did not reflect momentary changes in effort allocation within the primary task. The distribution of recognition as a function of time of presentation of the words reflected a retention curve with a strong recency effect, indicating some shortterm but very little long-term memory for the secondary message. The same curves were obtained in both dual-task conditions and in the control condition. These findings are similar to findings obtained in dichotic listening experiments for the nonshadowed material (e.g., Norman, 1969). It should be noted that, although the recognition rates for the first three 
critical words in the dual-task conditions in the present experiment were lower than in the control condition, the recognition rates were always higher than the false alarm rate, indicating some processing of the left-ear message.

The lack of an effect of momentary processing load of the primary task along with the failure of the secondary task to produce a significant decrement on the primary task, ${ }^{1}$ and a relatively low recognition for the secondary message, suggest that the recognition task did not receive active attention. An active strategy of attending in which allocation of attention is controlled by feedback from performance (Kahneman, 1973,1975 ) might be abandoned as too costly when the system is already meeting heavy demands. This could be the case in the present experiment. Both the primary and the secondary tasks were auditory shortterm memory tasks, and together they might result in overload. A secondary task which places less load on short-term memory may encourage the subject to attempt a more active strategy toward it, producing moment-by-moment competition between the primary and secondary tasks. The next experiment employed such a secondary task: Subjects searched for a target word in the left-ear message.

\section{EXPERIMENT 2}

\section{Method}

Subjects. Sixteen students, nine females and seven males aged 20-25 years, participated in the experiment. All subjects were native speakers of Hebrew, were right-handed, and had no hearing problems.

Primary tak. The primary task was the same as in Experiment 1.

Secondary task. The secondary task involved searching for a "known target" in the word series presented to the left ear. After each completion of the primary task, the subject indicated whether the Hebrew word "kadur" (ball) had appeared in the left-ear message. The target actually appeared on $80 \%$ of the trials in each of the dual-task conditions and in the secondarytask control condition. The target word appeared equally often at each of the following times (although the subjects were not aware of this): with the first or third digit in the listening phase, the word "answer," or the second digit in the response phase.

Payoff matrix. The subjects were paid .06 Israeli pounds for correct performance on the primary task and .03 Israeli pounds for a correct detection or a correct rejection when the target word was not present. They were penalized .15 Israeli pounds for reporting a target when it had not been presented. The reward for a correct response in the detection task was contingent on success on the primary task.

Design and Procedure. The design and procedure were the same as in Experiment 1.

\section{Results}

Performance on the primary task. Mean percentages of success on the primary task are presented in Table 1. A three-way analysis of variance (task $x$ condition $\times$ subjects) indicated a significant difference between the repeat and the transform task $[F(1,15)=68.66, p<.001]$. The difference between dual-task and control conditions just approached significance $[F(1,15)=4.187, .05<\mathrm{p}<.10]$. The interaction between task and condition was not significant $(\mathrm{F}<1)$.

Mean percentages of failure on the primary task in the dual-task conditions as a function of the time of occurrence of the target word are plotted in Figure 2. A three-way analysis of variance (task $\times$ time $\times$ subjects) was performed. The effect of task $[F(1,15)=$ $56.63, p<.001]$ and the effect of time $[F(3,45)=$ $4.63, \mathrm{p}<.01]$ were significant. The interaction between task and time $(F<1)$ was not significant. Thus, the time of occurrence of the target had an effect on the repeat and the transform performance.

Failures on the primary task in the dual-task conditions are apparently due to the event of detecting the target word rather than to monitoring for a target: when a target was not presented in the dualtask conditions, subjects' performance $(93.33 \%$ success on the repeat task; $73.63 \%$ on the transform task) did not differ from their performance in the control condition. These data confirm prior demonstrations of no decrement due to divided attention in the ability to monitor for a target, unless a judg-

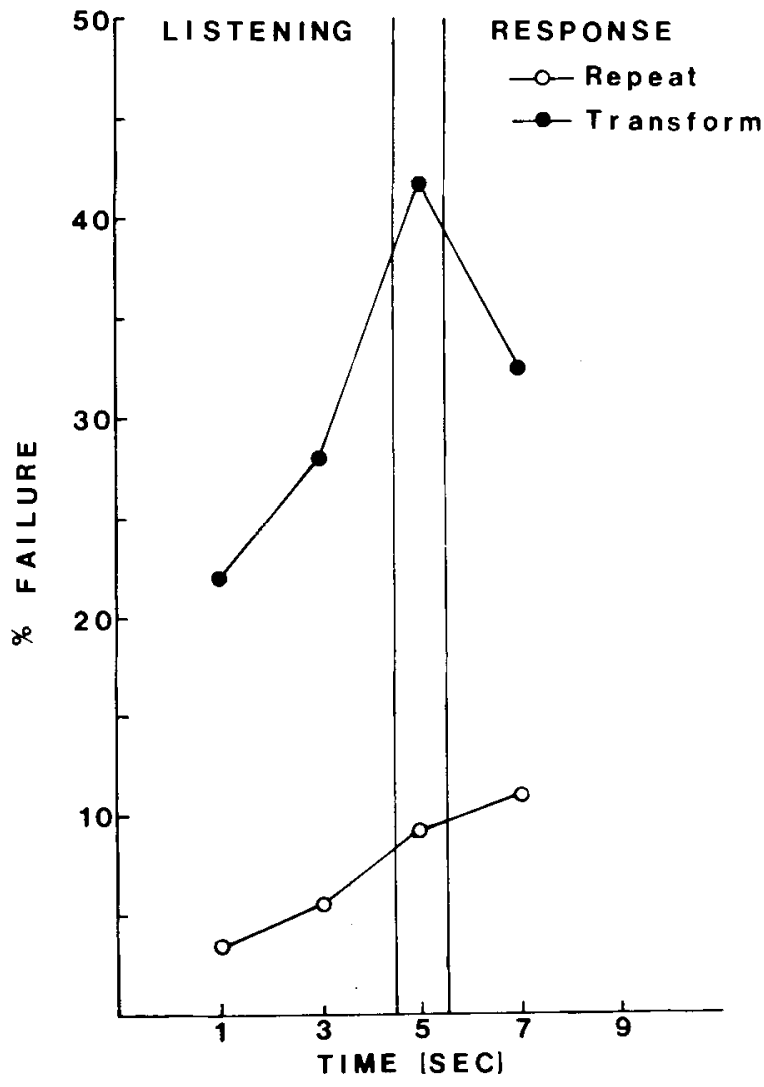

Figure 2. Reaults of Experiment 2: Mean percentage of failure on the primary task as a function of time of presentation of the target word. 
ment was made that a target had occurred (e.g., Duncan, 1980; Moray, 1975; Sorkin, Pohlmann, \& Woods, 1976). Granted that the effort distribution in the primary task followed an inverted-U curve, more interference from detection of a target was expected in the middle of the primary task. Since there was only one time point in the response phase, the appropriate comparisons rather than a trend analysis were performed. The tests showed no significant difference between performance on the repeat task when the target appeared in the middle of the task and when it appeared in the listening phase or in the response phase. The detection of a target did cause more interference to the transform task when the target was presented at the middle of the task (simultaneously with the word "answer") than when it was presented at the beginning of the task $[F(1,45)=$ $4.50, p<.05]$.

Performance on the secondary task. Mean percentages of failure on the detection task as a function of the time at which the target was presented are plotted in Figure 3.

The analysis of detection performance in the dualtask condition included only trials on which the primary task was correctly performed. A three-way analysis of variance (condition $\times$ time $\times$ subjects) indicated that the effect of condition $[F(2,30)=14.17$, $p<.001]$, the effect of time $[F(3,45)=3.45, p<.025]$, and the interaction between condition and time $[F(6,90)=2.28, p<.05]$ were significant. NewmanKeuls tests revealed that the subjects' failure on the detection task was higher when they simultaneously

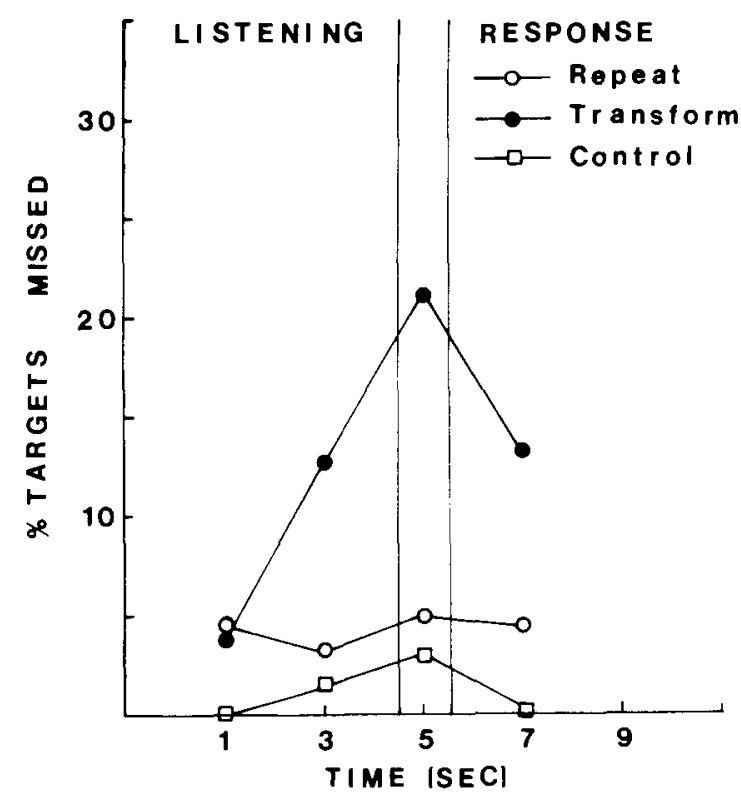

Figure 3. Results of Experiment 2: Mean percentage of failure in detection as a function of time of presentation of the target word. performed the transform task than when they performed the repeat task $[q(2,15)=4.70, p<.01]$, and that their detection performance on the dual-task conditions was impaired relative to that on the control condition $[q(3,15)=5.88, p<.01$, for the transform task; $q(2,15)=4.66, p<.01$, for the repeat task]. Subjects were less successful at detecting the target when it occurred at the middle of the transform task than when it occurred at the beginning of it $[F(1,45)=$ $6.39, \mathrm{p}<.025$ ]. (This analysis was performed on the "difference scores.") Subjects never reported a target when it had not been presented.

Detection performance was sensitive to betweentask variation in processing demands, and, in the transform-task condition, detection performance was also sensitive to within-task variation. The lack of sensitivity to within-task variation in the repeat-task condition might be due to the relatively small attentional demands of the present detection task. It is reasonable to assume that subjects were able to recognize the target without full semantic processing, perhaps on the basis of the word's acoustic characteristics. A detection task which requires full semantic analysis is expected to be more demanding (Kahneman, 1973) and thus more vulnerable to interference. The next experiment employed such a secondary task: Subjects searched for a member of semantic category occurring at critical times during the primary task.

\section{EXPERIMENT 3}

\section{Method}

Subjects. Six females and six males between the ages of 21 and 28 participated in the experiment. All subjects were native speakers of Hebrew, were right-handed, and had no hearing problems.

Primary task. The primary task was the same as in the previous experiments.

Secondary task. The secondary task involved searching for a member of a semantic category in the word series presented to the left ear. For each block, the subject was given a semantic category and was instructed to report at the completion of the primary task which member of the target category had been included in the left-ear message. Each category appeared twice, once in a repeat block and once in a transform block. The categories were: animals, professions, flowers, chemical elements, fruits, countries, furniture, Israeli towns, capital cities, and birds. All trials in each of the dual-task conditions and in the secondary-task control condition included a target word. A target word appeared equally often at the same times as the "known target" in the preceding experiment, and also simultaneously with the last digit spoken by the subject.

Payoff matrix. The subject was paid .06 Israeli pounds for a correct performance on the primary task and .03 Israeli pounds for a correct report of the target word, but only if the primary task was successfully performed.

Dealgn and Procedure. The design and procedure were the same as in the previous experiments.

\section{Results}

Performance on the primary task. Mean percentages of success on the primary task are presented in 


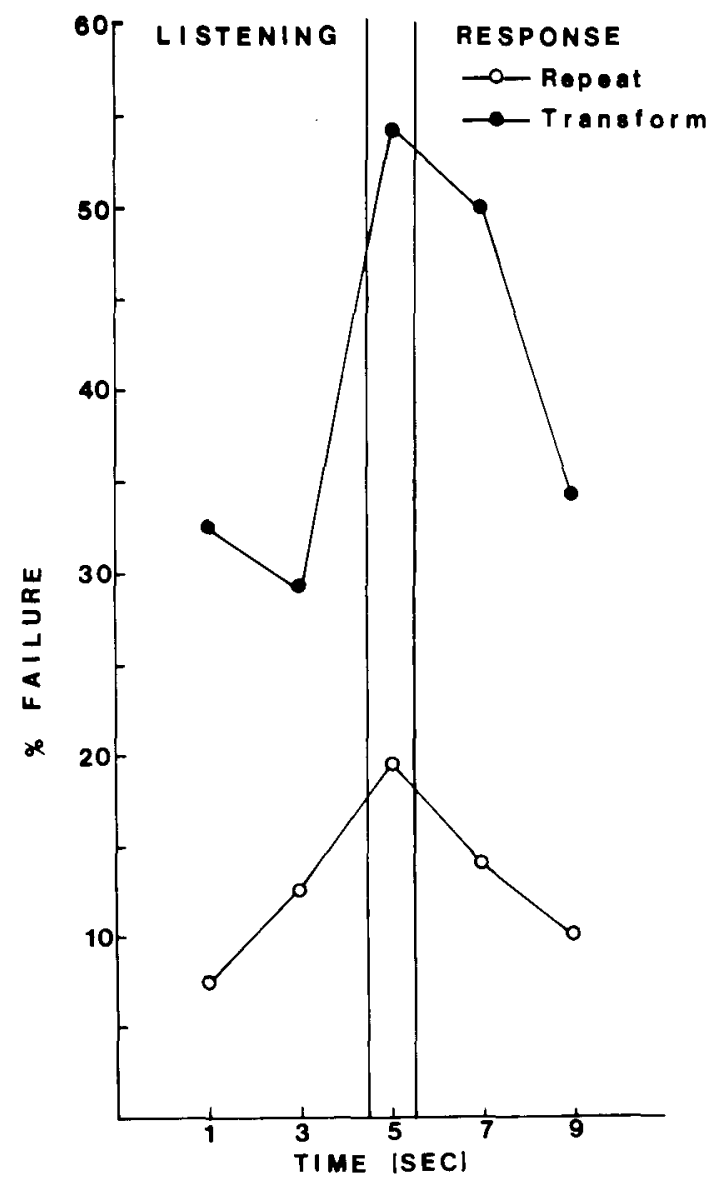

Figure 4. Results of Experiment 3: Mean percentage of fallure on the primary task as a function of time of presentation of the turget word.

Table 1. A three-way analysis of variance (task $\times$ condition $\times$ subjects) indicated a significant difference between the repeat and the transform task $[F(1,11)=$ 53.04, $\mathrm{p}<.001$ ], a significant difference between dual-task and control conditions $[F(1,11)=14.31$, $\mathrm{p}<.01$ ], and no significant interaction between task and condition $(F<1)$. These results suggest that the primary task was affected by the demand to perform the secondary task.

Figure 4 shows mean percentages of failure on the primary task as a function of the time at which the target word was presented. Analysis of variance (task $x$ time $\times$ subjects) revealed that the effect of task $[F(1,11)=121.21, p<.001]$ and the effect of time $[F(4,44)=6.97, \mathrm{p}<.001]$ were significant. The interaction between task and time was not significant $(F<1)$. The quadratic regression of the failure as a function of time of presentation of the target was significant $[F(1,11)=22.56, p<.001]$, demonstrating more interference from the detection task when the target word occurred at the middle of the task (either repeat or transform) than when it occurred at the beginning of the listening phase or at the end of the response phase.
Performance on the secondary task. Mean percentages of failure on the detection task are plotted in Figure 5.

The analysis of the detection performance on dualtask conditions included only trials on which the primary task was correctly performed. A three-way analysis of variance (conditions $\times$ task $\times$ subjects) indicated that the effect of condition $[F(2,22)=7.33$, $\mathrm{p}<.01]$, the effect of time $[\mathrm{F}(4,44)=5.09, \mathrm{p}<.01]$, and the interaction between condition and time $[F(8,88)=3.61, p<.01]$ were significant. NewmanKeuls tests revealed again that the subjects were less successful in detecting the target when they performed the transform task than when they performed the repeat task $[q(2,11)=4.07, p<.05]$, and that their detection performance on the dual-task conditions was impaired relative to the control condition $[q(3,11)=4.68, p<.05$, for the transform task; $q(2,11)=1.27, p>.05$, for the repeat task, not significant but in the same direction]. In order to examine the distribution of failure on detection as a function of time of presentation, a trend analysis was performed on the "different scores"; the quadratic regression was significant $[F(1,11)=24.69, p<.001]$ and suggested an inverted- $U$ curve.

Primary-/secondary-task interference was greater in the present experiment than in Experiment 2. Primarytask performance was lower in the dual-task conditions of the present experiment than in the pre-

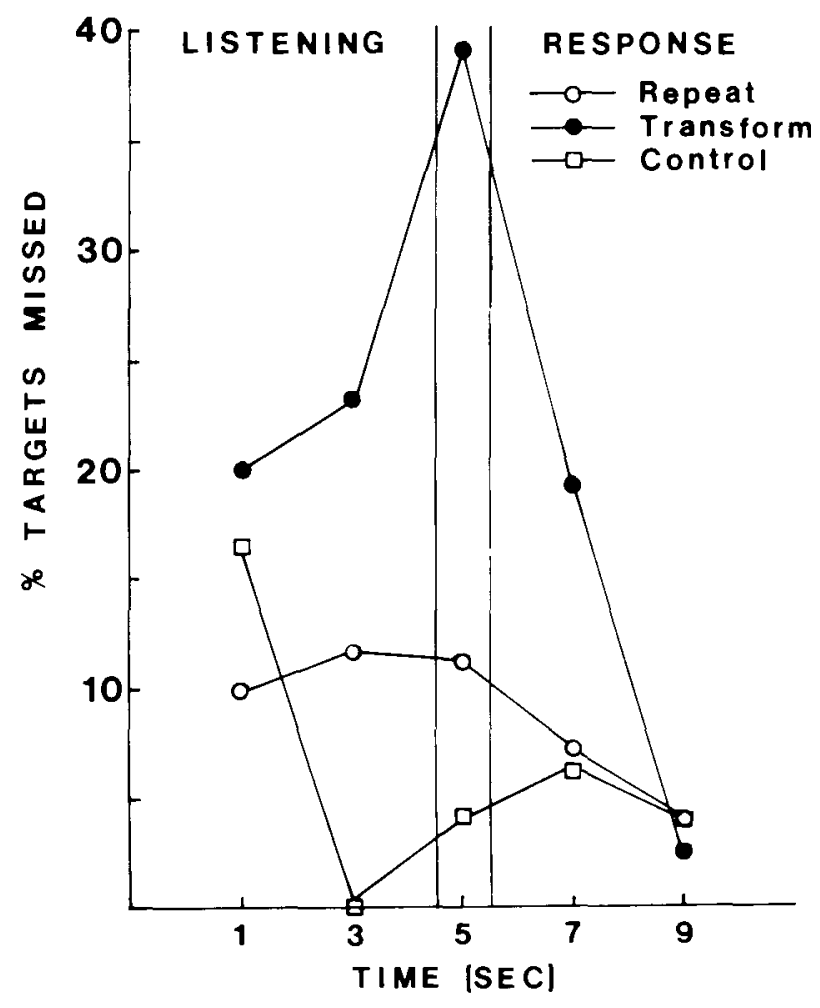

Figure 5. Results of Experiment 3: Mean percentage of fallure In detection as a function of time of presentation of the target word. 
vious experiment $[t(26)=2.75, p<.01$, for the repeat task; $t(26)=2.12, p<.025$, for the transform task]. Detection performance was also lower in the dual-task conditions of the present experiment than in the previous experiment, especially under the transform condition $[t(26)=2.17, p<.025]$ (the analysis was done on the "difference scores" for each experiment). These results indicate that detection by semantic category was, indeed, more demanding than detection of acoustic target, and they confirm previous studies that show that the further into the system information passes prior to selection, the greater is the capacity required to focus on the target (Johnston \& Heinz, 1978).

An interesting finding in the present experiment is the low level of performance on the transform task, even in the control condition. Performance on the transform task both in the dual-task condition and in the control condition differed significantly from performance on the same task in Experiment 1 [ $t(22)=$ $3.17, \mathrm{p}<.05$, for the dual-task condition; $t(22)=$ $1.76, \mathrm{p}<.05$, for the control condition]. The difference in the control conditions cannot be attributed to the differences in the ability of the two groups of subjects to perform the transform task, since the two groups did not differ from each other in the practice trials of the primary task. Furthermore, the two experiments were replicated, and exactly the same pattern of results was found. Logan (1978) proposed that, in a search task, attention is allocated to preparation for the task as a whole as well as to the execution of individual mental operations upon stimulus presentation. The role of attention in preparation suggests maintenance of a divided set (although biased towards the primary task) in the dual-task trials in the present experiment. Since, within blocks, the control trials were mixed with dual-task trials, it seems reasonable to assume that the subjects did not switch completely from a divided set on these trials.

\section{GENERAL DISCUSSION}

In all experiments, the transform task interfered with the secondary task more than did the repeat task, indicating that transformation imposed an additional processing load during multiple-item presentation. These results are in accordance with previous studies that have shown, using physiological measures, a higher level of activity during performance of the transform task than of the repeat task (Kahneman \& Beatty, 1966; Kahneman et al., 1969) and support the conclusion from other studies that mental transformation requires processing capacity (Kerr, 1973).

The results of Experiments 2 and 3 also showed an effect of momentary processing load of the primary task. More primary-/secondary-task interference occurred when the target appeared at times of maxi- mum effort in the primary task as has been found in previous studies using both physiological measures (Kahneman \& Beatty, 1966; Kahneman et al., 1969) and behavioral measures (for the transform task, Kahneman, 1970; Kahneman et al., 1967). This pattern of momentary competition between the two activities was more prevalent when the primary task and/or the secondary task were more demanding (i.e., transform vs. repeat and semantic vs. acoustic detection).

Thus, when the secondary task involved monitoring for and detecting a designated target word (Experiments 2 and 3 ), detection performance was sensitive to between-task and within-task variation in processing demands, and the interference between the tasks was mutual. However, when the secondary task involved listening to a list of words for later recognition (Experiment 1), recognition performance in the dual-task conditions was low relative to the control condition, but there was no mutual, momentby-moment competition between the primary and the secondary tasks. The different patterns of interference suggest different allocation policies. With the detection tasks, both the primary and the secondary tasks were actively attended to. In this case, allocation of attention to concurrent activities is expected to vary from moment to moment, depending on the attentional demands of both tasks (Kahneman, 1973, 1975). On the other hand, with the recognition task, the secondary task was passively attended to and the allocation of attention was concerned only with the demands of the primary task. It was suggested that subjects adopted a passive strategy toward the recognition task because this task places more load on short-term memory and, together with the primary task, might cause overload. An alternative interpretation attributes the difference in attentional strategies to the nature of the secondary tasks. The detection tasks provided a definite target, thus allowing a channeling of effort for achievement of the goal of the task, while the recognition task did not. Also, research in the area of memory which has studied recognition suggests that a recognition test involves less effortful processing than, for example, a recall test (Hasher \& Zacks, 1979; Kintsch, 1977). The present findings suggest that a recognition test that involves a more passive mode of retrieval might increase the probability of a passive mode of attending to the to-be-recognized list of words, at least under conditions of load.

Experiments in divided attention using the dichotic listening paradigm also suggest different allocation policies when the task involves search and detection vs. listening for later recognition. When the task was identification of a target (with immediate or delayed response), subjects have had no difficulty in searching for a target item in two dichotic lists, but there have been negative correlations between the proba- 
bilities of detecting simultaneous target items (Duncan, 1980; Kahneman, 1975; Moray, 1975; Moray \& Fitter, 1973; Moray et al., 1976). On the other hand, when the task was listening to long dichotic lists of words for later recognition, recognition performance was lower than in focused attention, but there was no negative correlation between the probabilities of recognizing simultaneous items (Kahneman, 1975). With shorter lists, recognition performance improved and manifested a negative correlation between recognition probabilities of simultaneous items, although this correlation was lower than for recall probabilities of simultaneous items in lists of the same length (Kahneman, 1975).

Although the present experiments were not designed to test the hypothesis of multiple resources, the convergence of the findings of Experiments 2 and 3 , in which the primary and the secondary tasks shared the same auditory modality, with the findings of previous experiments in which separate modalities were employed between tasks (i.e., auditory and visual, Kahneman et al., 1967; Kahneman, 1970) does not seem to support the idea of independent, modality-specific resources (e.g., Treisman \& Davis, 1974; Wickens, 1980).

Altogether, the present findings support the central notion of capacity theory: When the demands that two concurrent activities impose on a common pool of capacity increase, the interference between the tasks also increases. However, different secondary tasks can produce different attentional demands or attentional strategies, thus influencing the ability of the secondary task to track fine processing demands of the primary task.

\section{REFERENCES}

Bataur, R. [Basic word list for elementary schools.] Tel-Aviv: Otsar Ha-Moreh, 1968. (In Hebrew)

BEATTY, J. Task-evoked pupillary response, processing load, and the structure of processing resources. Psychological Bulletin, 1982, 91, 276-292.

Brondbent, D. E. Perception and communication. London: Pergamon Press, 1958.

Brondbent, D. E. Decision and stress. London: Academic Press, 1971.

Deut8ch, J. A., \& Deut8ch, D. Attention: Some theoretical considerations. Psychological Review, 1963, 70, 80-90.

DeuTsch, J. A., \& Deutsch, D. Comments on "Selective attention: Perception or response?" Quarterly Journal of Experimental Psychology, 1967, 19, 362-363.

Duncan, J. The locus of interference in the perception of simultaneous stimuli. Psychological Review, 1980, 87, 272-300.

HAshrR, L., \& ZACKs, R. T. Automatic and effortful processes in memory. Journal of Experimental Psychology: General, 1979, 108, 356-388.

Johnston, W. A., \& Heinz, S. P. Flexibility and capacity demands of attention. Journal of Experimental Psychology: General, 1978, 107, 420-435.

Kahneman, D. Remarks on attention control. Acta Psychologica, 1970, 33, 118-131.

Kahneman, D. Attention and effort. Englewood Cliffs, N.J: Prentice-Hall, 1973.

Kahneman, D. Effort, recognition and recall in auditory atten- tion. In P. M. A. Rabbit \& S. Dornic (Eds.), Attention and performance $V$. New York: Academic Press, 1975.

Kahneman, D., \& BeatTy, J. Pupil diameters and load on memory. Science, 1966, 154, 1583-1585.

Kahneman, D., Beatty, J., \& Pollack, I. Perceptual deficit during a mental task. Science, 1967, 157, 218-219.

Kahneman, D., Tursky, B., Shapiro, D., \& Crider, A. Pupillary, heart rate and skin resistance changes during a mental task. Journal of Experimental Psychology, 1969, 79, 164-167.

KeEle, S. W. Attention and human performance. Pacific Palisade, Calif: Goodyear, 1973.

KerR, B. Processing demands during mental operations. Memory \& Cognition, 1973, 1, 401-412.

Kintsck, W. Memory and recognition. New York: Wiley, 1977.

LogAN, G. D. Automatic processing in search tasks. Journal of Experimental Psychology: General, 1978, 107, 32-63.

Moray, N. Where is capacity limited? A survey and a model. Acta Psychologica, 1967, 27, 84-92.

Moray, N. A data base for theories of selective listening. In P. M. A. Rabbitt \& S. Dornic (Eds.), Attention and performance $V$. London: Academic Press, 1975.

Moray, N., \& FitTer, M. A theory and measurement of attention. In S. Kornblum (Ed.), Attention and performance $V I$. New York: Academic Press, 1973.

Moray, N., Fitter, M., Ostry, D., Favteau, D., \& Nagy, V. Attention to pure tones. Quarterly Journal of Experimental Psychology, 1976, 28, 271-283.

Navon, D., \& Gopher, D. On the economy of the human processing system. Psychological Review, 1979, 86, 214-255.

Navon, D., \& Gophen, D. Task difficulty, resources, and dualtask performance. In Reymond S. Nickerson (Ed.), Attention and performance VIII. Hillsdale, N. J: Erlbaum, 1980.

Norman, D. A. Memory while shadowing. Quarterly Journal of Experimental Psychology, 1969, 21, 85-94.

Norman, D. A., \& Boвrow, D. G. On data-limited and resourcelimited processes. Cognitive Psychology, 1975, 7, 44-56.

Posner, M. I., \& SNyder, C. R. R. Attention and cognitive control. In R. L. Solso (Ed.), Information processing and cognition: The Loyola Symposium. Hillsdale, N.J: Erlbaum, 1975.

Sorkin, R. D., Pohlmann, L. E., \& Wood, D. L. Detection interaction between auditory channels. Perception \& Psychophysics, 1976, 19, 290-295.

Treigman, A. M. Strategies and models of selective attention. Psychological Review, 1969, 76, 282-299.

Tre isman, A., \& Davis, A. Divided attention between eye and ear. In S. Kornblum (Ed.), Attention and performance IV. New York: Academic Press, 1973.

Wickens, C. D. The structure of attentional resources. In S. Nickerson (Ed.), Attention and performance VIII. Hillsdale, N.J: Erlbaum, 1980.

\section{NOTE}

1. The present results are nearly significant. However, this experiment was replicated twice. One replication was identical to the present experiment. The other involved a change in the payoff matrix in order to increase the subjects' motivation to perform the recognition task. A reward for a correct recognition was again contingent on successful performance on the primary task, but subjects received rewards only for correctly recognizing at least four words, and not for successful performance on the primary task. This change in the payoffs did not change the pattern of the results. In both replications, the pattern of the results was identical to that of the present experiment: Performance on the secondary task was not sensitive to momentary changes in processing demands within the primary task, and the secondary task produced no significant decrements on the primary task.

(Manuscript received April 4, 1982; revision accepted for publication September 13, 1982.) 